\title{
Differences in Somatosensory Function During Premenopause and Early and Late Postmenopause in Patients With Burning Mouth Syndrome
}

\author{
Kana Ozasa \\ Nihon University \\ Noboru Noma ( $\square$ noma.noboru@nihon-u.ac.jp ) \\ Nihon University \\ Kosuke Watanabe \\ Nihon University \\ Andrew Young \\ University of the Pacific \\ Eli Eliav \\ University of Rochester Medical \\ Yoshiki Imamura \\ Nihon University
}

\section{Research Article}

Keywords: Burning mouth syndrome, Quantitative sensory testing, Menopause, 40 Visual Analogue Scale

Posted Date: April 20th, 2021

DOI: https://doi.org/10.21203/rs.3.rs-421114/v1

License: () (1) This work is licensed under a Creative Commons Attribution 4.0 International License.

Read Full License 
1 Differences in somatosensory function during premenopause and early and late

2 postmenopause in patients with burning mouth syndrome

3 Kana Ozasa $^{1} \cdot$ Noboru Noma $^{1,2 *} \cdot$ Kosuke Watanabe $^{1} \cdot$ Andrew Young $^{3} \cdot$ Eli Eliav $^{4} \cdot$

4 Yoshiki Imamura ${ }^{1,2}$

$5 \quad{ }^{1}$ Department of Oral Diagnostic Sciences, Nihon University School of Dentistry, Tokyo,

6 Japan

$7 \quad{ }^{2}$ Clinical Research Division, Dental Research Institute, Nihon University, Tokyo, Japan

$8{ }^{3}$ Department of Diagnostic Sciences, Arthur Dugoni School of Dentistry, University of

9 the Pacific, San Francisco, United States

$10 \quad{ }^{4}$ Eastman Institute for Oral Health, University of Rochester Medical

11 Center, Rochester, NY, USA

\section{*Corresponding Author}

Dr. Noboru Noma DDS, PhD

14 Author contributions

15 Department of Oral Diagnostic Sciences, Nihon University School of Dentistry, and

16 Division of Clinical Research, 1-8-13, Kandasurugadai, Chiyoda-ku, Tokyo 101-8310,

17 Japan

18 Tel: 81-3-3219-8099; Fax: 81-3-3219-8346 
19 Email: noma.noboru@nihon-u.ac.jp

20 ABSTRACT

21 Background: This study aimed to examine the association between somatosensory 22 dysfunction and burning mouth syndrome (BMS) in premenopausal, early 23 postmenopausal, and late postmenopausal patients, using a standardized Quantitative 24 Sensory Testing (QST) protocol, and to determine the predictive value of thermal or 25 mechanical perception by QST for detecting BMS.

26 Methods: The study included 36 female participants with BMS (12 premenopausal, 10

27 early postmenopausal, and 14 late postmenopausal) and 42 age- and sex- matched healthy 28 volunteers (21 premenopausal, 10 early postmenopausal, and 11 late postmenopausal).

29 Neurophysiological tests were used to evaluate somatosensory dysfunction at the tongue.

30 Results: Z-scores in the late postmenopausal BMS group revealed a gain of function for

31 the cold pain threshold and heat pain threshold $(\mathrm{Z}=2.08$ and 3.38 , respectively). In the multiple regression analysis with the Visual Analogue Scale as the dependent variable, the vibration detection threshold predicted severity of burning mouth sensation in the

34 premenopausal group.

Conclusions: Late postmenopausal patients with BMS showed an increased response of

36 the tongue to noxious thermal stimuli. This supports the theory that changes in sex 
37 hormones may affect trigeminal somatosensory function in particular, during the late

38 postmenopausal stage in patients with BMS.

39

40 KEYWORDS: Burning mouth syndrome, Quantitative sensory testing, Menopause,

41 Visual Analogue Scale

42

43

44

45

46

47

48

49

50

51

52

53

54 


\section{Background}

56 Burning mouth syndrome (BMS) (also known as glossodynia, glossopyrosis, oral 57 dysesthesia, or stomatodynia) is chronic oral mucosal pain or discomfort, usually with a burning quality, that is not caused by any other condition or disease [1]. Women are affected 3 to 20 times more frequently than men, usually at menopausal or postmenopausal ages. The character of BMS condition is described as numbing, stinging

61 and pain [2].

62 The World Health Organization (WHO) defines menopause, also known as

64 climacteric, as the permanent cessation of menstruation resulting from the loss of ovarian follicular activity. Amenorrhea must occur for 12 consecutive months before natural menopause is recognized [3, 4]. During menopause, women experience a variety of predictable symptoms and conditions related to changes in sex hormone levels and aging. It typically occurs between 49 and 52 years of age [5]. receptors for sex steroidal hormones, similar to those located in the genital epithelium, are also present in the oral mucosa $[6,7]$. During the female lifetime, distinct changes in 
73 estrogen and progesterone activities occur several times. These steroids are

74 neuroprotective and are involved in the synthesis of neurotransmitters that generate pain-

75 related behaviors. The serum hormonal imbalance during menstruation, pregnancy, and

76 the perimenopausal period can result in numerous systemic and local symptoms,

77 including a burning sensation in the oral mucosa [7]. More recently, an enticing

78 hypothesis has been proposed that BMS is associated with an alteration of gonadal,

79 adrenal, and neuroactive steroid levels. Woda et al surmised that reduced synthesis of

80 ovarian steroids after menopause induces deficiency or dysfunction in adrenal steroids,

81 reducing the neuroprotective effects of steroids on neural tissues [8]. The predilection of

82 BMS in post-menopausal women also suggests reduced levels of sex hormones, which

83 can influence somatosensory function, may be a factor [9-11]. Among patients with BMS, a high preponderance in menopausal women suggests that nociception in the mouth is particularly sensitive to modulation by ovarian hormone levels [12]. A study in ovariectomized female rats showed an increased

88 sensitivity to nociceptive stimulation in the orofacial region [13]. However, clinical

89 behavioral studies have not verified this in premenopausal or early and late postmenopausal patients with BMS. 
93 Quantitative sensory testing (QST) studies of the pain threshold of patients with BMS

94 have had similar findings. Yilmaz et al. found that patients with BMS were more sensitive

95 to cold hyperalgesia than patients with lingual nerve impairment [15]. Grushka et al.

96 reported that heat pain tolerance was significantly decreased in patients with BMS [16].

97 QST alterations are not exclusive to BMS. De Kruijf et al. reported a significant association between years after menopause and cold and warmth sensitivity thresholds in patients with chronic pain [17]. In a previous study, we found an association between somatosensory dysfunction and disease duration in patients with BMS [14, 18].

We aimed to assess the somatosensory function in pre-menopausal and early and late postmenopausal patients with BMS, compared with healthy volunteers, and to investigate the association between QST (Quantitative Sensory Testing) data and stage of menopause. In addition, we investigated the predictive value of thermal and mechanical parameters for detecting the severity of burning sensation. 


\section{Participants and Methods}

111 This study was approved by the Ethical Committee of Nihon University School of

112 Dentistry ((EP16 D020-1; Feb.19.2020) and was conducted in accordance with the

113 Helsinki Declaration. Informed consent of all patients and volunteers was mandatory. All

114 patients were recruited by the same trained examiner (NN). Systemic diseases as well as

115 participants who had used pain medication, antidepressants, or non-steroidal anti-

116 inflammatory drugs during the previous 1 month were excluded.

The following definitions were used to determine post-menopausal status in this

119 study: Women who had a regular period in the past three months were categorized as premenopause. Women whose last menstrual period occurred $>12$ months prior were categorized as postmenopausal. Postmenopausal women who reached menopause $\leqslant 5$ years prior were classified as the early post-menopausal group, while those who had reached menopause $>5$ years prior were classified as the late postmenopausal group [19]. 
127 postmenopausal BMS $(n=10)$, and late postmenopausal BMS $(n=14)$. Likewise, the

128 healthy volunteers were divided into three groups: premenopausal control $(n=21)$, early

129 postmenopausal control $(n=10)$, and late postmenopausal control $(n=11)$.

130

131 QST was performed at the tip of the tongue. BMS was diagnosed according to

132 the International Classification of Headache Disorders, Third Edition [20]. All

133 participants were examined in a quiet, temperature-controlled room $\left(20-23^{\circ} \mathrm{C}\right)$. The

134 presence of intraoral spontaneous pain was assessed in patients with BMS using the

135 Visual Analogue Scale (VAS). For the VAS, each patient was shown a paper on which a

136 100-mm-long line was drawn, marked at one end with 0 and at the other end with 100;

137 the patients were asked to pointed to the position on the line that indicated the degree of

138 pain they were feeling at the first visit, considering 0 as the absence of pain and 100 as

139 the highest pain.

140

141 The QST protocol

142 The QST protocol used in this study was based on that of the German Research Network

143 on Neuropathic Pain [21]. 
146 Cold detection threshold (CDT), warmth detection threshold (WDT), thermal sensory

147 limen (TSL), cold pain threshold (CPT), and heat pain threshold (HPT) were determined

148 by using a computer-controlled Peltier - type thermode (stimulation area $16 * 16 \mathrm{~cm}^{2}$,

149 Somedic ${ }^{\circledR}$ Sales AB, Hörby, Sweden) and the Classic Method of Limits. The baseline

150 temperature was set to $32^{\circ} \mathrm{C}$, and temperature modulation was performed between 5 and

$15150^{\circ} \mathrm{C}$. CDT and WDT were represented as the temperature difference from $32^{\circ} \mathrm{C}$ for non-

152 noxious warm or cold temperature changes. The first painful cold (CPT) or hot (HPT)

153 sensation was recorded, and TSL was determined by alternating warm and cold stimuli.

154 The investigators instructed the patients to press a button as soon as they noticed the

155 required sensation. Subjects had to keep their eyes closed during the entire QST procedure.

156 Three repetitive measurements were taken to reduce bias, and thresholds were represented

157 as means.

158

\section{Mechanical tests}

160 The mechanical QST protocol used in this study was comprised of tests for the mechanical

161 detection threshold (MDT), mechanical pain threshold (MPT), mechanical pain

162 sensitivity (MPS), dynamic mechanical allodynia (DMA), wind-up ratio (WUR), 
163 vibration detection threshold (VDT), and pressure pain threshold (PPT). For a full

164 discussion of the protocol, see [21]. MDT was investigated with a standardized set of

165 modified von Frey filaments (Optihair2®-Set, Marstock Nervtest, Germany) and using

166 the "Method of Limits" with forces between 0.125 and $512 \mathrm{mN}$ graded by a factor of 2

167 (1-2 s contact time). For MPT, custom-made weighted pinprick stimuli with a flat contact

168 area of 0.25 diameter were used. Five repetitive measurements were performed for MDT

169 and MPT and presented as the geometric mean. MPS was measured using pinprick stimuli

170 and calculated as the geometric mean of all numerical ratings. DMA was assessed by

171 using soft stimuli with a cotton wisp exerting a force of $\sim 5 \mathrm{mN}$, a cotton wool tip fixed to

172 an elastic strip $(\sim 100 \mathrm{mN})$, and a cotton wool tip fixed to a stiff strip $(\sim 200-400 \mathrm{mN})$.

173 WUR was evaluated by applying repeated painful stimuli (single pinprick stimulus 128

$174 \mathrm{mN}$ compared with a series of 10 repetitive pinprick stimuli of the same intensity). VDT

175 was investigated by applying a Rydel-Seiffer (Aesculap, Tuttlingen, Germany) graded

176 tuning fork $(64 \mathrm{~Hz}, 8 / 8$ scale) over the tongue in three stimulus repetitions.

178 Statistical analyses

179 Unpaired t test was used to determine the significance of any differences between the

180 QST results of premenopausal, early postmenopausal, and late postmenopausal patients 
181 with BMS and healthy controls. All data in the text and table are presented as

182 means \pm standard deviations (SD). To examine differences between patients with BMS and

183 controls in the QST variables, a Z-score transformation was performed for all QST

184 variables to provide a somatosensory profile. The detailed method of Z-score calculation

185 has been described elsewhere [22]. Briefly, Z-score $=($ single premenopausal patient with

186 BMS X - premenopausal control means) / premenopausal control SD, or (single early

187 postmenopausal patient with BMS X -early postmenopausal control means) / early post-

188 menopausal control SD, or (single late postmenopausal patient with BMS X -late post-

189 menopausal control means) / late post-menopausal control SD. A Z-score greater than

$190 \pm 1.96$ (i.e., outside the $95 \%$ confidence interval $[\mathrm{CI}]$ ) was considered an abnormal value.

191 A positive Z-score indicated sensory gain, and a negative Z-score indicated sensory loss.

192 Z-scores in the figures are presented as means \pm standard errors.

In addition, multiple regression analysis was performed to define the contribution of the independent variables such as thermal parameters (CDT, WDT, TSL

196 CPT, and HPT) and mechanical parameters (MDT, MPT, VDT, PPT, WUR, and MPS) to

197 the dependent variable (VAS: spontaneous pain) in the premenopausal, early postmenopausal, and late postmenopausal patients with BMS. All statistical analyses 
199 were performed using SPSS (ver. 27 for Windows; IBM, Tokyo, Japan). Significance 200 level was set at $p<0.05$ for all statistical tests.

\section{Results}

204 There was no significant difference in age between premenopausal patients with BMS 205 (40.1 \pm 6.0 years) and premenopausal controls (45.2 \pm 2 . years 4$)$, between early 206 postmenopausal patients with BMS (53.2 \pm 2.7 years) and early postmenopausal controls 207 (55.6 \pm 2.8 years), and between late postmenopausal patients with BMS $(70.1 \pm 5.0$ years) 208 and late postmenopausal controls $(64.9 \pm 10.8$ years $)$. The mean severity of oral mucosal 209 burning pain (spontaneous pain) in premenopausal, early postmenopausal, and late 210 postmenopausal patients with BMS (53.2 \pm 2.7 years) was $31.8 \pm 25.3,47.0 \pm 28.7$ and $211 \quad 52.7 \pm 32.5$, respectively. Table 1 shows the raw data at the tongue for all QST in the premenopausal, early postmenopausal, and late postmenopausal BMS groups. Likewise, QST raw data in the

215 premenopausal, early postmenopausal, and late postmenopausal control groups are 216 showed in Table 2. 
PPT was significantly decreased in the premenopausal BMS group compared to

221 significantly decreased in the BMS group compared with controls $(p<0.05)$. In the late and HPT between controls and patients with BMS, indicating cold and heat hyperalgesia at the tongue $(p<0.01)$ (Table 1$)$.

\section{Z-score}

227 In the premenopausal and early postmenopausal BMS groups, the means of all the parameters on the tongue remained within the $95 \% \mathrm{CI}$ of the baseline reference database (Z-scores within \pm 1.96 ; Fig. 1a and Fig. 2a). Of 12 patients with BMS in the premenopausal group, abnormal Z-scores at the tongue were detected for WDT in two patients, TSL in three patients, CPT in two patients, MDT in one patient, MPT in one patient, VDT in one patient, PPT in seven patients, WUR in one patient, and MPS in one patient. (Fig. 1b). Of 10 patients in the early postmenopausal BMS group, abnormal Z- 
one patient, CPT in two patients, MPT in two patients, VDT in one patient, PPT in one patient, and MPS in two patients (Fig. 2b). mean gain of function by CPT and HPT at the tongue ( $\mathrm{Z}=2.08$ and 3.38, respectively). Of patients. (Fig. 3b).

\section{Regression analysis}

246 A stepwise multiple regression analysis was performed with spontaneous pain as the

247 dependent variable. The model using mechanical parameters (MDT, MPT, VDT, PPT,

248 WUR, MPS) explained $17.1 \%$ of variance in spontaneous pain in premenopausal patients

249 with BMS $\left[\operatorname{adj} . \mathrm{R}^{2}=0.58 ; \mathrm{F}=3.56 ; p=0.025\right]$. VDT contributed significantly to the model, while the other parameters did not (Table 3). There was no evidence of the occurrence of DMA, which was not included in the parameters as an independent variable. 
254 significantly predictive for spontaneous pain in premenopausal patients with BMS [adj.

$\left.255 \mathrm{R}^{2}=0.03 ; \mathrm{F}=0.93 ; p=0.30\right]$. When analyzed for the early postmenopausal BMS group, the

256 model was not predictive when the predictor variables were mechanical $\left[\operatorname{adj} . \mathrm{R}^{2}=0.32\right.$;

$257 \mathrm{~F}=0.63 ; p=0.45]$ or thermal $\left[\operatorname{adj} . \mathrm{R}^{2}=0.55 ; \mathrm{F}=0.36 ; p=0.79\right]$. Similarly, when analyzed for 258 the late postmenopausal BMS group, the model was not predictive when the predictor 259 variables were mechanical $\left[\operatorname{adj} . \mathrm{R}^{2}=0.17 ; \mathrm{F}=0.68 ; p=0.76\right]$ or thermal $\left[\operatorname{adj} . \mathrm{R}^{2}=0.38\right.$; 260 $\mathrm{F}=2.64 ; p=0.06]($ Table 4$)$

\section{Discussion}

In the present study, the QST results for CPT and HPT at the tongue were significantly more sensitive in the BMS group than in the control group at the late postmenopausal stage. In a previous QST study, De Kruijf et al. found a significant association between years after menopause and cold and warmth sensitivity thresholds [17]. One possible reason for the increased CPT may be that drastic menopausal changes induce a significant reduction in neuroprotective steroids. This reduction in neuroprotective and neuroregenerative capacities may preferentially put small A $\delta$ fibers at a risk [23]. Another 
271 possible mechanism is that neuroactive steroids may directly or indirectly affect cold

272 sensitivity in postmenopausal patients with BMS. Yilmaz et al. and Hartmann et al. also

273 reported cold hyperalgesia in patients with BMS but not in patients with lingual nerve 274 impairment $[15,24]$.

277 response of the tongue to cold pain stimulation (CPT Z-score=2.08). A $\delta$ cold afferents 278 seem to be impaired more often than $\mathrm{C}$ fibers in patients with BMS, indicating an 279 imbalance in the small fiber input to the central nervous system [15]. Zagury et al. 280 reported an extended painful aftersensation following cold application in atypical 281 odontalgia patients compared with control subjects [25]. This suggests a neuropathic 282 mechanism involving central factors. The cold test may be a diagnostic marker for central 283 sensitization in patients with chronic pain, such as BMS. Several histological studies related to cold hypersensitivity have also been reported [26,27]. Possible mechanisms that underlie the cold hypersensitivity in chronic pain may include increased expression and

286 activity of the transient receptor potential ankyrin 1 (TRPA1) receptors on trigeminal sensory neurons [28]. The BMS mouse model showed overexpression of both TRPV1 and TRPA1 receptors in the trigeminal ganglion [29]. Recent animal studies also 
289 demonstrated low incidences of transient receptor potential melastatin 8 (TRPM8) and

290 TRPV1 co-expression in trigeminal ganglion neurons [30]. Thus, cold pain might be

291 mediated via the activation of TRPM8-expressing polymodal nociceptor cold receptors.

292 The modulation of the interaction of TRPV1, TRPA1 receptors, and TRPM8 in trigeminal

293 ganglion neurons may contribute to cold hyperalgesia.

294

Late post-menopausal patients with BMS also showed an increased response of the tongue to heat pain stimulation (HPT Z-score=3.38). Grushka et al. demonstrated that

297 patients with BMS were significantly less tolerant of heat pain on the tongue than control

298 subjects [16]. It is well known that the psychophysical responses to noxious thermal

299 stimuli are dependent not only on stimulus intensity, but also duration of stimulus,

300 interstimulus interval, and characteristics of prior conditioning stimuli. It is possible that

301 duration testing for heat pain tolerance may cause sensitization of the $\mathrm{C}$ fiber polymodal

302 nociceptors and A $\delta$ heat nociceptors in patients with BMS [30]. This sensitization may

303 account for the decreased heat pain tolerance of patients with BMS. 
cutaneous cells and neurons, glia, and Schwann cells in the peripheral and central nervous

309 thermal nociceptive signals from the orofacial regions.

312 which may occur more readily with higher temperatures and longer durations. Shinozaki et al. proposed that the mechanism of pain habituation observed during the repetition of a noxious stimulus is explained by $\mathrm{C}$ - fiber function, and lack of this phenomenon may

315 be associated with impaired function of $\mathrm{C}$ fibers [33]. Recently, we demonstrated in another study that BMS is associated with a deficit in inhibitory conditioned pain modulation [34]. The pain inhibitory system was impaired when the $47^{\circ} \mathrm{C}$ conditioning stimulus was applied to the hand in patients with BMS [34]. It is possible that this pain inhibitory impairment may have developed due to the lack of habituation of pain sensation when applying noxious thermal stimulation to the hand. This may be associated with the increased response to thermal nociceptive stimuli, which may result in heat hyperalgesia. In this study, we also investigated the relationship between QST parameters and 
325 parameters can be used as an early sign of BMS development. Multiple regression analysis using mechanical parameters (MDT, MPT, VDT, PPT, WUR, and MPS) as

327 predictive variables for spontaneous pain explained $17.1 \%$ of the variance in early stage of patients with BMS, although VDT alone was found to be significant. Additionally, in 329 the premenopausal stage, PPT at the tongue was significantly lower in the BMS group than in the control group. of-onset of menopause displayed greater painful and non-painful mechanical sensitivity than patients with late age-of-onset of menopause [34]. In fact, prevalence of orofacial pain with migraine or temporomandibular joint disorders seems to be much higher during peak reproductive years $[35,36]$. Hormonal changes associated with pre-menopause or early menopause may modulate pain hypersensitivity. Thus, ovarian hormones may exert their effect on somatosensory brain activity by altering opiate neurotransmitters [38]. To the best of our knowledge, this is the first study to demonstrate that QST can generate objective information (specifically the VDT) that aids in the early detection of BMS in premenopausal patients. Treatment for BMS is more effective when introduced 
early [39]. Therefore, the early detection and timely referral to an orofacial pain specialist

344 are critical and may reduce the chronic pain and associated cost. vary according to the ovulatory phase of the menstrual cycle, may affect sensory changes in the masticatory muscle, abdomen, and lower back areas in healthy women. In this study,

349 we did not determine the phase of the menstrual cycle in healthy volunteers. This may 350 have affected the QST results. Further studies are needed to determine whether a 351 relationship exists between QST data and progesterone and estrogen levels. Also, in the late postmenopausal group, HPT and TSL trended towards significance in the multiple regression analysis ( $p=0.055$ and $p=0.05$ respectively; constant $p=0.06$ ). It is possible that the small sample size lacked the power to detect significant predictive value for those thermal tests.

\section{Conclusions}

358 A standardized battery of QSTs demonstrated that an early transition to menopause may influence mechanical pain sensitivity, while late menopause may influence thermal pain sensitivity. Overall, our findings suggest that changes in sex hormones may directly or 
361 indirectly affect trigeminal somatosensory function during the postmenopausal stage in 362 patients with BMS.

367 CDT - Cold detection threshold, thermal sensory limen (TSL)

368 CPT - Cold pain threshold

369 DMA - Dynamic mechanical allodynia

370 HPT - Heat pain threshold

371 MDT - Mechanical detection threshold

372 MPS - Mechanical pain sensitivity

373 MPT - Mechanical pain threshold

$374 \quad$ PPT - Pressure pain threshold QST - Quantitative Sensory Testing

376 TRPA1 - Transient receptor potential ankyrin 1

377 TRPM8 - Transient receptor potential melastatin 8

378 VAS - Visual Analogue Scale 
379 VDT - Vibration detection threshold

380 WDT - Warmth detection threshold

381 WUR - Wind-up ratio

382

383

\section{$384 \quad$ Declarations}

- Ethics approval and consent to participate

386

387

388

from all patients and volunteers.

389

- Consent for publication

$390 \quad$ Not applicable.

391

- Availability of data and materials

392

The datasets used and analysed during the current study are available from the

393

corresponding author on reasonable request. 
The authors declare that they have no competing interests.

This study was supported in part by research grants from the Sato and Uemura are for clinical data collection. AY, EE and Y. I have reviewed the article. All the authors have read approve the final manuscript. conflicts of interest related to this study. 
410 Kana Ozasa, Email: deka18006@g.nihon-u.ac.jp

411 Noboru Noma, Email: noma.noboru@nihon-u.ac.jp

412 Kosuke Watanabe, Email: watanabe.k.0824@gmail.com

413 Andrew Young, Email: ayoung@pacific.edu

414 Eli Eliav, Email: Eli_Eliav@urmc.rochester.edu

415 Yoshiki Imamura, Email: imamura.yoshiki@nihon-u.ac.jp

416

\section{References}

4181 Jääskeläinen SK, Woda A. Burning mouth syndrome. Cephalalgia. 2017;37(7):627-47.

419 doi: 10.1177/0333102417694883. Epub 2017 Mar 15.

4202 Imamura Y, Shinozaki T, Okada-Ogawa A, Noma N, Shinoda M, Iwata K, et al. An 421 updated review on pathophysiology and management of burning mouth syndrome with 422 endocrinological, psychological and neuropathic perspectives. J Oral Rehabil. $423 \quad 2019 ; 46(6): 574-87$.

4243 Research on the menopause in the 1990s. Report of a WHO Scientific Group. World 425 Health Organ Tech Rep Ser. 1996;866:1-107.

4264 Munro MG. Southern California Permanente Medical Group's Abnormal Uterine 
427 Bleeding Working Group. Investigation of women with postmenopausal uterine bleeding:

428 clinical practice recommendations. Perm J. 2014;18(1):55-70. doi: 10.7812/TPP/13-072

4295 Takahashi TA, Johnson KM. Menopause. Med Clin North Am. 2015;99(3):521-34. doi:

$430 \quad$ 10.1016/j.mena.2015.01.006.

4316 Meurman JH, Tarkkila L, Tiitinen A. The menopause and oral health. Maturitas.

432 2009;20;63(1):56-62. doi: 10.1016/j.maturitas.

4337 Friedlander AH. The physiology, medical management and oral implications of 434 menopause. J Am Dent Assoc. 2002;133(1):73-81. Review.

4358 Woda A, Dao T, Gremeau-Richard C. Steroid dysregulation and stomatodynia (burning 436 mouth syndrome). J Orofac Pain. 2009;23(3):202-10.

4379 Meurman JH, Tarkkila L, Tiitinen A. The menopause and oral health. Maturitas $438 \quad 2009 ; 63(1): 56-62$.

43910 Friedlander AH. The physiology, medical management and oral implications of 440 menopause. J Am Dent Assoc. 2002;133(1):73-81.

44111 Aryeh HB, Gottlieb I, Ish-Shalom S, David A, Szargel H, Laufer D. Oral complaints 442 related to menopause. Maturitas. 1996;24(3):185-9.

44312 International Classification of Orofacial Pain, 1st edition (ICOP). [No authors listed] 444 Cephalalgia. 2020;40(2):129-221. 
44513 Pajot J, Ressot C, Ngom I, Woda A. Gonadectomy induces site-specific differences in 446 nociception in rats. Pain. 2003;104(1-2):367-73.

44714 Imamura Y, Okada-Ogawa A, Noma N, Shinozaki T, Watanabe K, Kohashi R, et al. A 448 perspective from experimental studies of burning mouth syndrome. J Oral Sci. $449 \quad 2020 ; 28 ; 62(2): 165-9$. 15 Yilmaz Z, Egbuniwe O, Renton T. The detection of small-fiber neuropathies in burning 451 mouth syndrome and iatrogenic lingual nerve injuries: use of quantitative sensory testing. 452 J Oral Facial Pain Headache. 2016;30(2):87-98. doi: 10.11607/ofph.1531.

45316 Grushka M, Sessle BJ, Howley TP. Psychophysical assessment of tactile, pain and 454 thermal sensory functions in burning mouth syndrome. Pain. 1987;28(2):169-84.

45517 de Kruijf M, Peters MJ, C Jacobs L, Tiemeier H, Nijsten T, Hofman A, et al. 456 Determinants for quantitative sensory testing and the association with chronic 457 musculoskeletal pain in the general elderly population. Pain Pract. 2016;16(7):831-41.

45818 Watanabe K, Noma N, Sekine N, Takanezawa D, Hirota C, Eliav E, Imamura Y. 459 Association of somatosensory dysfunction with symptom duration in burning mouth 460 syndrome. Clin Oral Investig. 2019;23(9):3471-7. 19 G K P, Arounassalame B. The quality of life during and after menopause among rural women. J Clin Diagn Res. 2013;7(1):135-9. 
20 IHS (2018) ICHD-3 The International Classification of Headache Disorders 3rd

edition. Int Headache Soc 38(1):1-211.

21 Rolke R, Baron R, Maier C, Treede DR, Beyer A, Binder A, et al. Quantitative sensory

testing in the German Research Network on Neuropathic Pain (DFNS): standardized

protocol and reference values. Pain. 2006;123(3):231-43.

468

22 Rolke R, Magerl W, Campbell KA, Schalber C, Caspari S, Birklein F, et al.

Quantitative sensory testing: a comprehensive protocol for clinical trials. Eur J Pain. 2006;10(1):77-88.

23 Jääskeläinen SK. Is burning mouth syndrome a neuropathic pain condition? Pain. 2018;159(3):610-3.

24 Hartmann A, Seeberger R, Bittner M, Rolke R, Welte-Jzyk C, Daubländer M.

Profiling intraoral neuropathic disturbances following lingual nerve injury and in burning mouth syndrome. BMC Oral Health. 2017;17(1):68. doi: 10.1186/s12903-017-0360-y. gingival cold allodynia: a novel finding in patients with atypical odontalgia. Oral Surg

478 Oral Med Oral Pathol Oral Radiol Endod. 2011;111(3):312-9. doi: 10.1016/j.tripleo.2010.10.008. Epub 2011 Jan 7. 
481 small-fiber sensory neuropathy causes burning mouth syndrome. Pain. 2005;115(3):332-

4827

48327 Borsani E, Majorana A, Cocchi MA, Conti G, Bonadeo S, Padovani A, et al. Epithelial 484 expression of vanilloid and cannabinoid receptors: a potential role in burning mouth 485 syndrome pathogenesis. Histol Histopathol. 2014;29(4):523-33.

487 Burning mouth syndrome as a trigeminal small fibre neuropathy: increased heat and 488 capsaicin receptor TRPV1 in nerve fibres correlates with pain score. J Clin Neurosci. 489 2007;14(9):864-71. Epub 2007 Jun 19.

49029 Elitt CM, Malin SA, Koerber HR, Davis BM, Albers KM. Overexpression of artemin 491 in the tongue increases expression of TRPV1 and TRPA1 in trigeminal afferents and 492 causes oral sensitivity to capsaicin and mustard oil. Brain Res. 2008;1230:80-90. doi: 10.1016/j.brainres.2008.06.119. Epub 2008 Jul 10. protein localization in trigeminal ganglion and taste papillae. Brain Res Mol Brain Res. 496 2005;136(1-2):91-8. Epub 2005 Mar 2. mechanisms of cutaneous hyperalgesia following mild injury by heat. J Neurosci. 
$1982 ; 2(6): 765-81$.

50032 Boucher Y, Simons CT, Carstens MI, Carstens E. Effects of gustatory nerve transection

501 and/or ovariectomy on oral capsaicin avoidance in rats. Pain. 2014;155(4):814-20.

50233 Shinozaki T, Imamura Y, Kohashi R, Dezawa K, Nakaya Y, Sato Y, et al. Spatial and 503 temporal brain responses to noxious heat thermal stimuli in burning mouth syndrome. $\mathrm{J}$

504 Dent Res. 2016;95(10):1138-46.

50534 Nishihara C, Watanabe K, Ozasa K, Khan J, Eliav E, Imamura Y, et al. Altered pain 506 modulation to noxious heat thermal stimuli in burning mouth syndrome. Oral Dis. $507 \quad 2020 ; 26(8): 1777-1782$.

50835 Martínez-Jauand M, Sitges C, Femenia J, Cifre I, González S, Chialvo D, et al. Age509 of-onset of menopause is associated with enhanced painful and non-painful sensitivity in 510 fibromyalgia. Clin Rheumatol. 2013;32(7):975-81.

51136 Schmidt PJ, Cardoso GM, Ross JL, Haq N, Rubinow DR, Bondy CA. Shyness, social 512 anxiety, and impaired self-esteem in Turner syndrome and premature ovarian failure.

$513 \quad$ JAMA. 2006;295(12):1374-6.

51437 Liao KL, Wood N, Conway GS. Premature menopause and psychological well-being.

515 J Psychosom Obstet Gynaecol. 2000;21(3):167-74.

51638 Martin VT. Ovarian hormones and pain response: a review of clinical and basic science 
517 studies. Gend Med. 2009;6 Suppl 2:168-92.

51839 Silvestre-Rangil J, Silvestre FJ, Tamarit-Santafé C, Bautista D. Burning mouth

519 syndrome: correlation of treatment to clinical variables of the disease. Med Oral Pathol $520 \quad$ Oral Cir Bucal. 2011;16(7):e890-4.

521

522

523

$524 \quad$ Figure Legends

525

526 Figla : Mean overall Z scores. Results of quantitative sensory testing (QST) (Z scores)

527 with BMS in the premenopausal group. All QST variables are presented as Z scores. A Z

528 score greater than 0 indicates increased sensation, and a $\mathrm{Z}$ score less than 0 indicates loss

529 of sensory function. $\mathrm{Z}$ scores greater than \pm 1.96 indicate values outside the $95 \%$

530 confidence interval of the baseline values. Abbreviations: cold detection threshold (CDT),

531 warmth detection threshold (WDT), thermal sensory limen (TSL), cold pain threshold

532 (CPT), heat pain threshold (HPT), mechanical detection threshold (MDT), mechanical

533 pain threshold (MPT), vibration detection threshold (VDT), pressure pain threshold (PPT),

534 wind-up ratio (WUR), and mechanical pain sensitivity (MPS). All data are presented 
as mean $\pm \mathrm{SE}$

536

537 Figlb : scatter plot with individual data point (premenopausal BMS patients).

538 Abbreviations: cold detection threshold (CDT), warmth detection threshold (WDT),

539 thermal sensory limen (TSL), cold pain threshold (CPT), heat pain threshold (HPT),

540 mechanical detection threshold (MDT), mechanical pain threshold (MPT), vibration

541 detection threshold (VDT), pressure pain threshold (PPT), wind-up ratio (WUR), and

542 mechanical pain sensitivity (MPS). All data are presented as mean $\pm \mathrm{SE}$.

543

544

545 Fig2a : Mean overall Z scores. Results of quantitative sensory testing (QST) ( $\mathrm{Z}$ scores)

546 with BMS in the early postmenopausal group. All QST variables are presented as Z scores.

547 A Z score greater than 0 indicates increased sensation, and a $\mathrm{Z}$ score less than 0 indicates

548 loss of sensory function. $Z$ scores greater than \pm 1.96 indicate values outside the $95 \%$

549 confidence interval of the baseline values. Abbreviations: cold detection threshold (CDT),

550 warmth detection threshold (WDT), thermal sensory limen (TSL), cold pain threshold

551 (CPT), heat pain threshold (HPT), mechanical detection threshold (MDT), mechanical

552 pain threshold (MPT), vibration detection threshold (VDT), pressure pain threshold (PPT), 
553 wind-up ratio (WUR), and mechanical pain sensitivity (MPS). All data are presented 554 as mean $\pm \mathrm{SE}$.

556 Fig2b : scatter plot with individual data point (the early postmenopausal patients).

557 Abbreviations: cold detection threshold (CDT), warmth detection threshold (WDT), 558 thermal sensory limen (TSL), cold pain threshold (CPT), heat pain threshold (HPT), 559 mechanical detection threshold (MDT), mechanical pain threshold (MPT), vibration detection threshold (VDT), pressure pain threshold (PPT), wind-up ratio (WUR), and mechanical pain sensitivity (MPS). All data are presented as mean $\pm \mathrm{SE}$.

Fig3a : Mean overall Z scores. Results of quantitative sensory testing (QST) (Z scores) with BMS in the late postmenopausal group. All QST variables are presented as Z scores.

566 A Z score greater than 0 indicates increased sensation, and a $\mathrm{Z}$ score less than 0 indicates loss of sensory function. $Z$ scores greater than \pm 1.96 indicate values outside the $95 \%$ confidence interval of the baseline values. Abbreviations: cold detection threshold (CDT), warmth detection threshold (WDT), thermal sensory limen (TSL), cold pain threshold (CPT), heat pain threshold (HPT), mechanical detection threshold (MDT), mechanical 
571 pain threshold (MPT), vibration detection threshold (VDT), pressure pain threshold (PPT),

572 wind-up ratio (WUR), and mechanical pain sensitivity (MPS). All data are presented

573 as mean $\pm \mathrm{SE}$.

574

575 Fig3b : scatter plot with individual data point (the late postmenopausal patients).

576 Abbreviations: cold detection threshold (CDT), warmth detection threshold (WDT),

577 thermal sensory limen (TSL), cold pain threshold (CPT), heat pain threshold (HPT),

578 mechanical detection threshold (MDT), mechanical pain threshold (MPT), vibration

579 detection threshold (VDT), pressure pain threshold (PPT), wind-up ratio (WUR), and

580 mechanical pain sensitivity (MPS). All data are presented as mean $\pm \mathrm{SE}$. 
Figures

\section{Premenopause BMS}
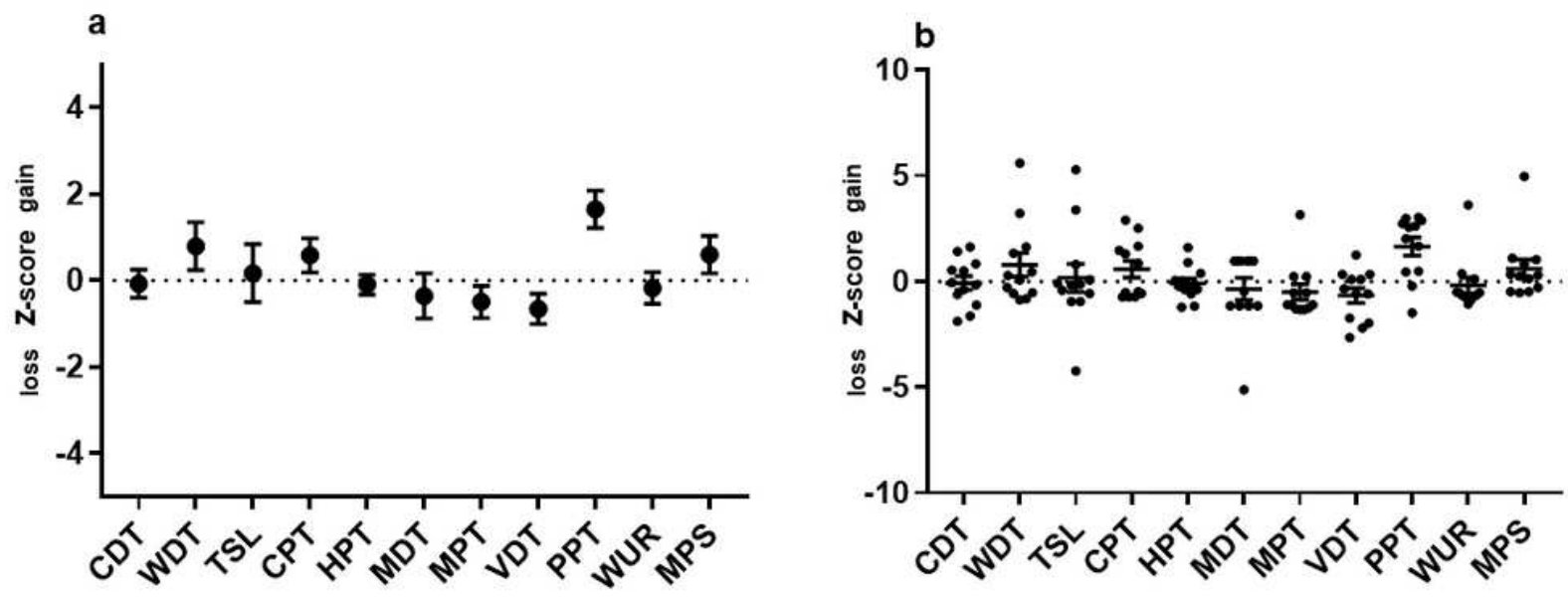

Figure 1

See the Manuscript Files section for the complete figure caption. 
The early postmenopause BMS
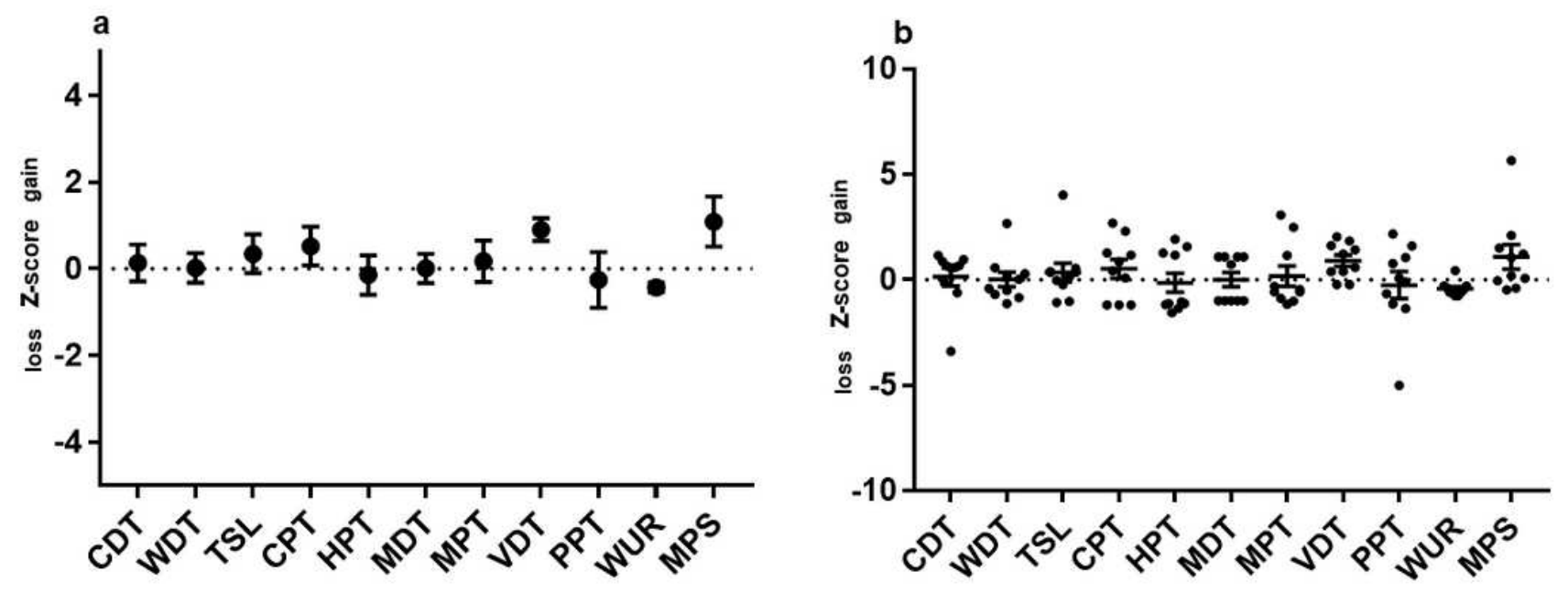

Figure 2

See the Manuscript Files section for the complete figure caption.

The late postmenopause BMS
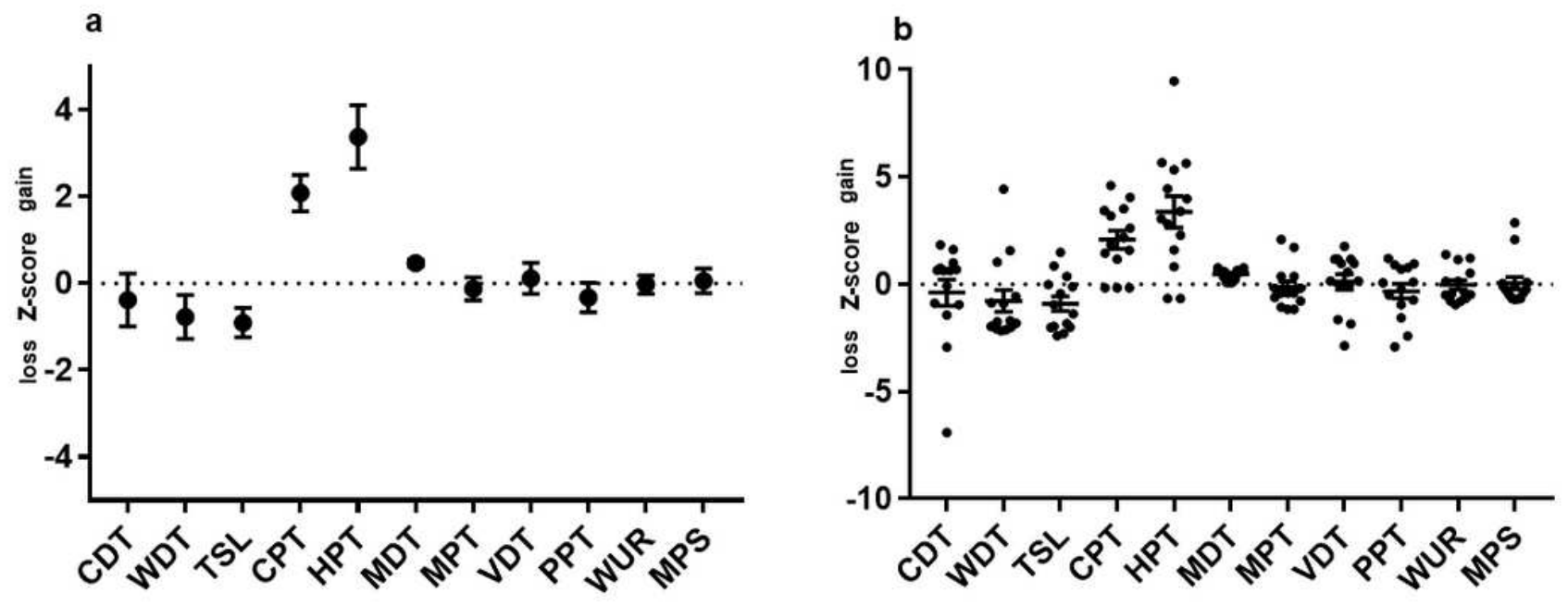
Figure 3

See the Manuscript Files section for the complete figure caption.

\section{Supplementary Files}

This is a list of supplementary files associated with this preprint. Click to download.

- Table1.tif

- Table2.tif

- Table3.tif

- Table4.tif 